\title{
Somatic intronic microsatellite loci differentiate glioblastoma from lower-grade gliomas
}

\author{
Enusha Karunasena ${ }^{1, *}$, Lauren J. McIver ${ }^{1, *}{ }^{,}$Brian R. Rood ${ }^{2}$, Xiaowei Wu ${ }^{3}$, Hongxiao \\ Zhu $^{3}$, Jasmin H. Bavarva ${ }^{1}$ and Harold R. Garner ${ }^{1}$ \\ ${ }^{1}$ Virginia Bioinformatics Institute, Medical Informatics and Systems Division; Blacksburg, VA \\ ${ }^{2}$ Center for Cancer and Blood Disorders at Children's National Medical Center; Washington, D.C \\ ${ }^{3}$ Department of Statistics at Virginia Tech; Blacksburg, VA \\ * These authors contributed equally to this work \\ Correspondence to: Harold R. Garner, email: garner@vbi.vt.edu \\ Keywords: GBM; microsatellite; glioma; oligodendroglioma; helicase; ubiquitin proteasome system \\ Received: March 3, $2014 \quad$ Accepted: June 4, $2014 \quad$ Published: June 5, 2014
}

This is an open-access article distributed under the terms of the Creative Commons Attribution License, which permits unrestricted use, distribution, and reproduction in any medium, provided the original author and source are credited.

\section{ABSTRACT}

Genomic studies of glioma sub-types have amassed new disease specific mutations, yet these only partially explain how mutations are linked to predisposition or progression. We hypothesized that microsatellite variation could expand the understanding of glioma etiology. Furthermore, germline markers for gliomas are typically undetectable; therefore we also hypothesize that the predictability of cancerassociated microsatellite loci in germline DNA may support the current hypothesis of a glioma cell of origin.

In this study, "normal" germline exome sequenced DNA from the 1000 Genomes Project $(n=390)$ were compared with exome sequences from germlines of subjects with WHO grade II and III lower-grade glioma (LGG, $n=136$ ) and WHO grade IV glioblastoma ( $G B M, n=252$ ) from The Cancer Genome Atlas to identify microsatellite loci non-randomly associated with glioma. From germline data, we identified 48 GBM-specific loci, 42 Lower-grade glioma specific loci and 29 loci that distinguish GBM from LGG ( $\leq \leq 0.01$ ). We then attempted to distinguish WHO grade II glioma $(n=67)$ from GBM resulting in 8 informative loci. Significantly, in all glioma grades, comparisons between tumor and matched germline sequences demonstrated no significant differences in these variants $(p \geq 0.01)$. Therefore, these microsatellite loci are considered to be components of grade-specific signatures for glioma which distinguish germline sequences of individuals with cancer from those of individuals that are "normal". In order to better understand the significance of these loci, we identified biological processes enriched in genes with these variants. Most strikingly, six helicase genes were enriched in the GBM cohort $\left(p \leq 1.0 \times 10^{-3}\right)$. The preservation of these glioma-specific loci could therefore serve as valuable diagnostic and therapeutic markers; especially since the heterogeneity of tumor cell populations can obscure the identification of mutations preceding a metastatic phenotype.

\section{INTRODUCTION}

Every year in the U.S. approximately 10,000 new adult malignant brain tumors are diagnosed[1] with the most common being glioblastoma (GBM; WHO grade IV astrocytoma)[2]. Treatment with radiation and chemotherapy yields a median survival of only 11-
14 months; therefore, GBM is considered a terminal diagnosis [3]. There are three main histological groups of adult gliomas: astrocytoma (A); oligodendroglioma (OD) which are slower-growing but rarely progress to GBM; and mixed glioma such as oligoastrocytomas (OA), a mix of A and $\mathrm{OD}[4,5]$. Astrocytoma is graded from I to IV according to the World Health Organization's (WHO) 


\begin{tabular}{|c|c|c|c|c|}
\hline & $\begin{array}{l}\text { Total MST Loci } \\
\text { (Exome Sequences) }\end{array}$ & $\begin{array}{l}\text { Significant MST Genotypes } \\
(p \leq 0.01)\end{array}$ & \begin{tabular}{|l|} 
FDR Corrected \\
CAML Genotypes \\
(Signature) \\
\end{tabular} & $\begin{array}{l}\text { Average Detectable Signature } \\
\text { Genotypes per Sample }\end{array}$ \\
\hline GBM & $26,021 \quad(\mathrm{SD} \pm 4,859)$ & 179 & 48 & $13.1( \pm 6.6)$ \\
\hline LGG & $26,596(\mathrm{SD} \pm 2,392)$ & 146 & 42 & $15.1( \pm 5.9)$ \\
\hline Grade II & $26,427(\mathrm{SD} \pm 2,333)$ & 75 & 8 & $4.2 \quad( \pm 1.7)$ \\
\hline
\end{tabular}

classification criteria while OD and OA are primarily in grades II and III. Lower grade adult astrocytomas can progress to higher grade tumors. Recurrence after therapy is common with $\mathrm{A}, \mathrm{OA}$, and most $\mathrm{OD}$ and is generally associated with more aggressive and infiltrative tumors [6].

High-throughput sequencing studies of tumor genomes have produced new molecular markers that have enhanced classification of GBM and highlighted molecular pathways that propagate pathogenesis and progression [6-9]. In this study, we have chosen to identify and characterize a specific component of glioma genomics- DNA microsatellite variants - a feature that is understudied relative to SNPs or epigenetic markers. Microsatellites (MST) are short tandem repeated sequences with variable lengths, making them excellent markers of disease[10]. Repetitive nucleotide sequences account for $55 \%$ of the intergenic region of the genome with $3 \%$ of these considered microsatellite repeats[10, 11]. Microsatellites can be found in both coding and non-coding regions, are generally 10-15 nucleotides in length and are found as monomers, di-, tri-, penta-, or hexamers[11, 12]. Expansion of tri-nucleotide repeat sequences are notably contributive to over 40 different neurological disorders including Fragile X, Huntington's and Parkinson's Disease[13]. These alterations are hypothesized to lead to changes in gene regulation through modified binding sites for transcription factors, splicing machinery, or microRNAs [10, 12, 13]. Thus, changes to microsatellite sequences elicit phenotypes and contribute to diseases, including cancer.

Therefore, we hypothesize that gliomagenesis is correlated with microsatellite variability at specific loci in germline DNA. Additionally, we demonstrate that germline (non-tumor) DNA is representative of the tumor and these glioma-specific microsatellite loci discriminate glioma grades (specifically II versus IV). With validation, such markers could be developed to aid clinical predictions. We further hypothesize that these loci could influence tumor biology, including a potential contribution to early glioma tumorigenesis.

\section{RESULTS}

\section{The two populations studied are GBM and lower- grade gliomas (LGG)}

Classifications were provided by The Cancer Genome Atlas (TCGA) and we have chosen to discuss our results using the same assemblage for scientific comparisons (as such, lower-grade gliomas are a mix of glioma types that are non-GBM samples). Microsatellite loci were identified from exome sequencing data for a cancer germline and a "normal" population. Those sequences that varied in length significantly between the two populations were compiled to create a 'signature' list (see Table 1). Signatures consist of variant allelic pairs (genotypes) from cancer-associated microsatellite loci (CAMLs). Three signatures are reported: GBM vs normals, LGG vs normals, and GBM vs LGG.

\section{Gliomas Compared with Normal Sample Sequences:}

The distribution of CAML genotypes found within samples differentiates the germlines of patients when compared to cancer-free controls. Considering the GBM signature, $19 \%$ of GBM germline samples had $\geq 75 \%$ of CAML genotypes and 16\% of GBM tumors also had $\geq 75 \%$ of these loci (Fig. 1A). Additionally, 100\% of the CAML genotypes from the GBM signature were discovered in $12 \%$ of GBM germline samples, while only $3 \%$ of "normal" samples demonstrated similar results. Also described in Figure 1 are normal samples compared with LGG: here the largest cluster of LGG germline samples $(20 \%)$ had $50 \%$ of the detectable CAML genotypes (Fig. 1B). In an alternative analysis, we removed Grade III astrocytomas ( $\mathrm{n}=28$; additionally, samples with non-descript pathology (16 described as NA and 2 as UNK)) to create a new LGG signature (see Fig. S3): this signature is composed of 24 CAMLs, of which all but 4 loci were in the LGG data that are described in Fig. 2B. Importantly, all 4 of these loci were identified 
as significant in the analysis associated with Figure 2B. Also, given the heterogeneous population in the LGG cohort, we isolated OD Grade II and Grade III ( $n=59)$ and show the distribution of LGG genotypes in these samples compared with the total LGG germline population $(\mathrm{n}=$ 92); the largest population of OD $(15 \%)$ had $25 \%$ of LGG CAMLs (see Fig. S3).
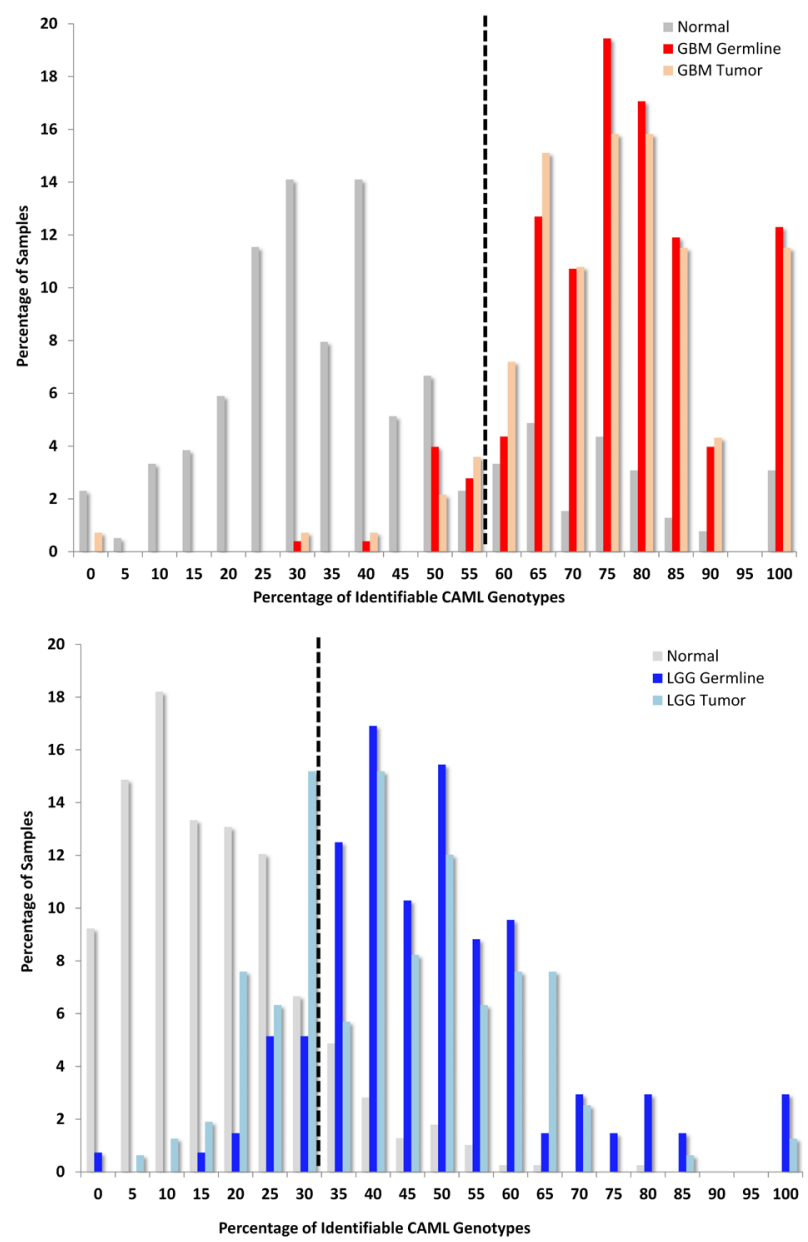

FIGURE 1: Variant Glioma Microsatellite Loci Significantly Differ from Normal Germlines. The y-axis describes the percentage of samples and the $\mathrm{x}$-axis shows a given fraction of detectable CAML genotypes; a receiver operator characteristic (ROC, see supplement) was used to determine the cut-off thresholds, which are 57\% GBM and 35\% LGG; illustrated by a black hashed-vertical line). (1A.) GBM: First, we compared TCGA GBM to an ethnically matched "normal" population in the 1000 Genomes Project ( $1 \mathrm{kGP})$ and calculated a sensitivity of $94 \%$ and specificity of $77 \%$ (corresponding GBM tumor sensitivity is $96 \%$ and specificity is $75 \%$ ). Second, we compared LGG to $1 \mathrm{kGP}$ and determined the sensitivity to be $91 \%$ and specificity at $86 \%$ (LGG tumor sensitivity is $84 \%$ and specificity is $86 \%$ compared with $1 \mathrm{kGP}$ ). (1B.) LGG: LGG encompassed WHO grade II and III A, OD, and OA. We identified the cut-off again using ROC analysis at $32 \%$. Sixty-six percent of LGG samples contained $\geq 40$ percent of LGG-specific CAML genotypes.

\section{Glioma Grades Compared with GBM Sequences:}

We compared LGG and GBM germline sequences (Fig 2A) to determine if there was information

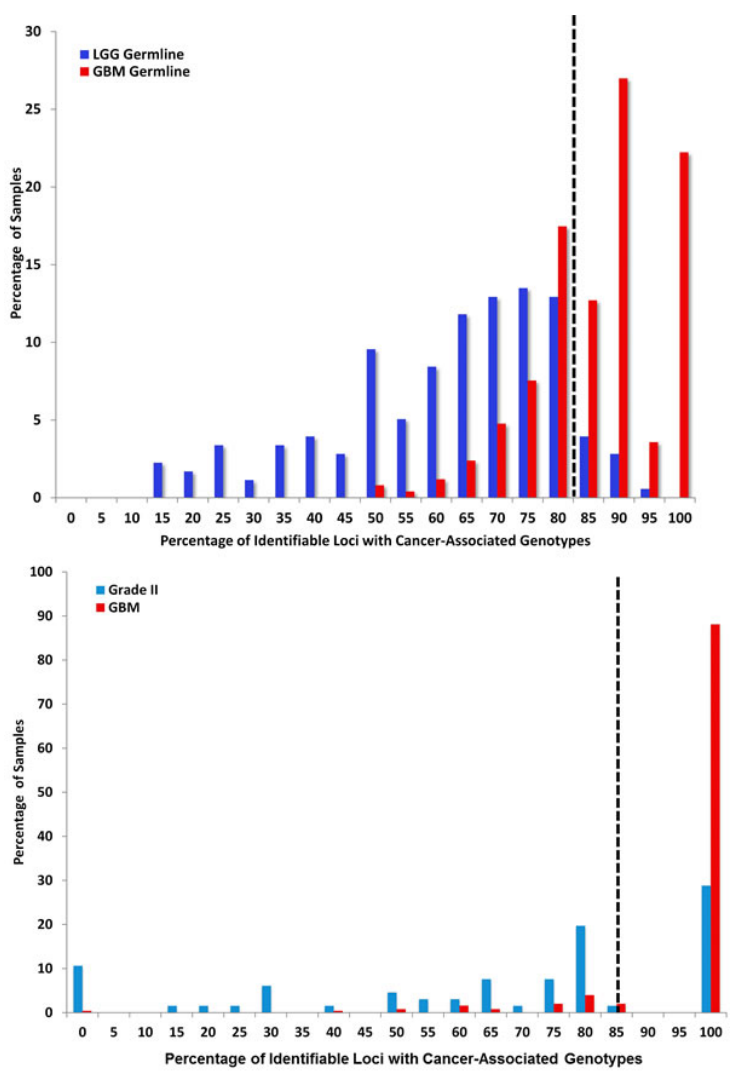

FIGURE 2: Variant Microsatellite Loci Distinguish Lower and High Grade Gliomas. (2A.) LGG versus GBM: CAML genotypes shared between LGG and GBM sequences were identified. GBM germline sequences contain $90 \%$ of the signature CAMLs, whereas $3 \%$ of $\mathrm{LGG}$ germlines were represented in the same group (2B.) Grade II versus GBM: Seventy-percent of Grade II A, OA, and some OD progress to Grade III or IV[40]. We identified Grade II glioma signature genotypes; we then measured the percentage of samples (GBM and Grade II) with these CAMLs. In this analysis we identified eight Grade II specific genotypes. The sensitivity of these loci is $90 \%$ and specificity is $70 \%$, with a cutoff of $85 \%$. GBM germline sequences shared an abridged population of LGG genotypes; $82 \%$ of identifiable Grade II germline genotypes were also found in GBM samples. (2C) Below $82 \%$, the percentage of CAML genotypes in LGG are more enriched. In Grade II samples, $\geq$ $75 \%$ of loci could be located in $26 \%$ of samples versus $4 \%$ of GBM. The genotypes identified in $19 \%$ of Grade II samples with $80 \%$ of CAMLs were located with the following genes (in order of significance): KIAA1219 (13 samples), SNX17 (12 samples), SACMIL (9 samples), MYCBP2 (8 samples), GFM1 (7 samples), COPS4 (6 samples), and CDC16 (1 sample). All eight signature loci were identifiable in the majority of Grade II, GBM, and the general population (1kGP; data not shown). We compared GBM with LGG germline and calculated sensitivity at $74 \%$ and specificity at $90 \%$ (tumor analysis shows sensitivity at $76 \%$ and specificity at $72 \%$ ). We identified a sensitivity of $90 \%$ and specificity of $70 \%$ for Grade II loci compared with GBM. 
Table 2: GBM and LGG Genes with CAMLs and Novel Transcript Isoforms. RNASeq data for GBM and LGG were screened for transcript diversity and abundance these were compared with normal adult brain transcript data, previously analyzed[16]. For GBM and LGG, described are genes for which an isoform was the most abundant transcript; reported are the average copies for these transcripts and the average copies of total transcripts for a gene. We also identified genes in which transcripts were exclusively identified in GBM and/or LGG compared with normal brain tissue. Ten CAMLs were shared between GBM and LGG, of these we identified three that were expressed in both sample populations; in both GBM and LGG the most abundant transcript for FUBP3 was an isoform compared with normal tissue which had one transcript that was a conserved sequence. RNASeq data from the Illumina BodyMap 2.0 Project was used to determine if transcripts had been identified for those genes which only showed expression in GBM or LGG in normal brain, all genes demonstrated positive expression in these data.

\begin{tabular}{|c|c|c|c|c|}
\hline \multicolumn{5}{|c|}{ GBM } \\
\hline Gene & Transcript Diversity & Avg. Copy of Gene & \multicolumn{2}{|c|}{ Avg. Copy of Novel Isoform } \\
\hline DICER1 (GBM) & 16 & 0.15 & \multicolumn{2}{|r|}{1.2} \\
\hline DICER1 (N) & 4 & 5.5 & & \\
\hline FGFR2 (GBM) & 11 & 0.24 & \multicolumn{2}{|r|}{1.6} \\
\hline FGFR2 (N) & 4 & 3 & & \\
\hline FUBP3 (GBM) & 5 & \multirow[t]{2}{*}{0.41} & \multicolumn{2}{|r|}{0.99} \\
\hline FUBP3 (N) & 1 & & & \\
\hline HYDIN (GBM) & 17 & \multirow[t]{2}{*}{0.48} & \multicolumn{2}{|r|}{3.45} \\
\hline HYDIN (N) & 1 & & & \\
\hline ICA1L (GBM) & 10 & \multirow[t]{2}{*}{2.4} & \multicolumn{2}{|r|}{0.41} \\
\hline ICA1L (N) & 3 & & & \\
\hline OFD1 (GBM) & 14 & \multirow[t]{2}{*}{0.18} & \multicolumn{2}{|r|}{0.86} \\
\hline OFD1 (N) & 1 & & & \\
\hline POLQ (GBM) & 7 & \multirow[t]{2}{*}{0.49} & \multicolumn{2}{|r|}{1.8} \\
\hline POLQ (N) & 1 & & & \\
\hline SPOPL (GBM) & 7 & \multirow[t]{2}{*}{1.04} & \multicolumn{2}{|r|}{0.24} \\
\hline SPOPL (N) & 2 & & & \\
\hline \multicolumn{5}{|c|}{ EXPRESSED ONLY IN GBM } \\
\hline Gene & Transcript Diversity & Avg. Copy of Gene & $\begin{array}{l}\text { Avg. Copy of } \\
\text { Novel Isoform }\end{array}$ & $\begin{array}{c}\text { Avg. Copy of } \\
\text { Common Transcript }\end{array}$ \\
\hline TTF2 & 10 & 0.29 & 1.03 & \\
\hline ACRC & 5 & 0.39 & 0.76 & 0.86 \\
\hline COL9A1 & 10 & 0.17 & 0.59 & \\
\hline CORIN & 6 & 0.09 & 0.02 & 0.92 \\
\hline FRMD7 & 1 & 5 & & \\
\hline GTPBP8 & 4 & 0.25 & 0.03 & 0.94 \\
\hline NSUN5 & 6 & 0.21 & 0.31 & 0.62 \\
\hline NUFIP1 & 4 & 0.29 & 0.09 & 0.98 \\
\hline RYR1 & 8 & 0.68 & 2.3 & \\
\hline SLC44A4 & 2 & 0.05 & 0.03 & 0.07 \\
\hline STRC & 8 & 0.03 & $\begin{array}{c}0.05 \\
0.005 \\
0.02\end{array}$ & 0.07 \\
\hline \multicolumn{5}{|c|}{ LGG } \\
\hline CDC16 (LGG) & 13 & 0.23 & 1.2 & \\
\hline CDC16(N) & 5 & 1.2 & & \\
\hline RBM5 (LGG) & 14 & 0.3 & 1.3 & \\
\hline RBM5 (N) & 5 & 2.6 & & 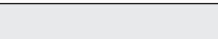 \\
\hline \multicolumn{5}{|c|}{ EXPRESSED ONLY IN LGG } \\
\hline $\mathrm{CDH} 16$ & 1 & 0.08 & & \\
\hline CDRT1 & 1 & 0.41 & & \\
\hline DENND3 & 13 & 0.64 & 0.65 & 2.2 \\
\hline LNX2 & 2 & 0.2 & 0.02 & 0.39 \\
\hline GUSB & 8 & 0.35 & 1.3 & \\
\hline ARSK & 5 & 0.1 & 0.01 & 0.38 \\
\hline & & KPRESSED IN GBM \& LGG & & \\
\hline EVC (GBM) & 8 & 0.24 & 0.76 & \\
\hline EVC (LGG) & 6 & 0.24 & 0.85 & 0.1 \\
\hline $\operatorname{EVC}(\mathrm{N})$ & 0 & & & \\
\hline FUBP3 (GBM) & 6 & 0.41 & 0.99 & \\
\hline FUBP3 (LGG) & 5 & 0.36 & 0.86 & \\
\hline FUBP3 (N) & 1 & 2 & & 2 \\
\hline PSME3 (GBM) & 6 & 0.29 & 0.24 & 0.93 \\
\hline PSME3 (LGG) & 11 & 0.15 & 0.09 & 0.77 \\
\hline PSME3 (N) & 1 & 3 & & \\
\hline
\end{tabular}



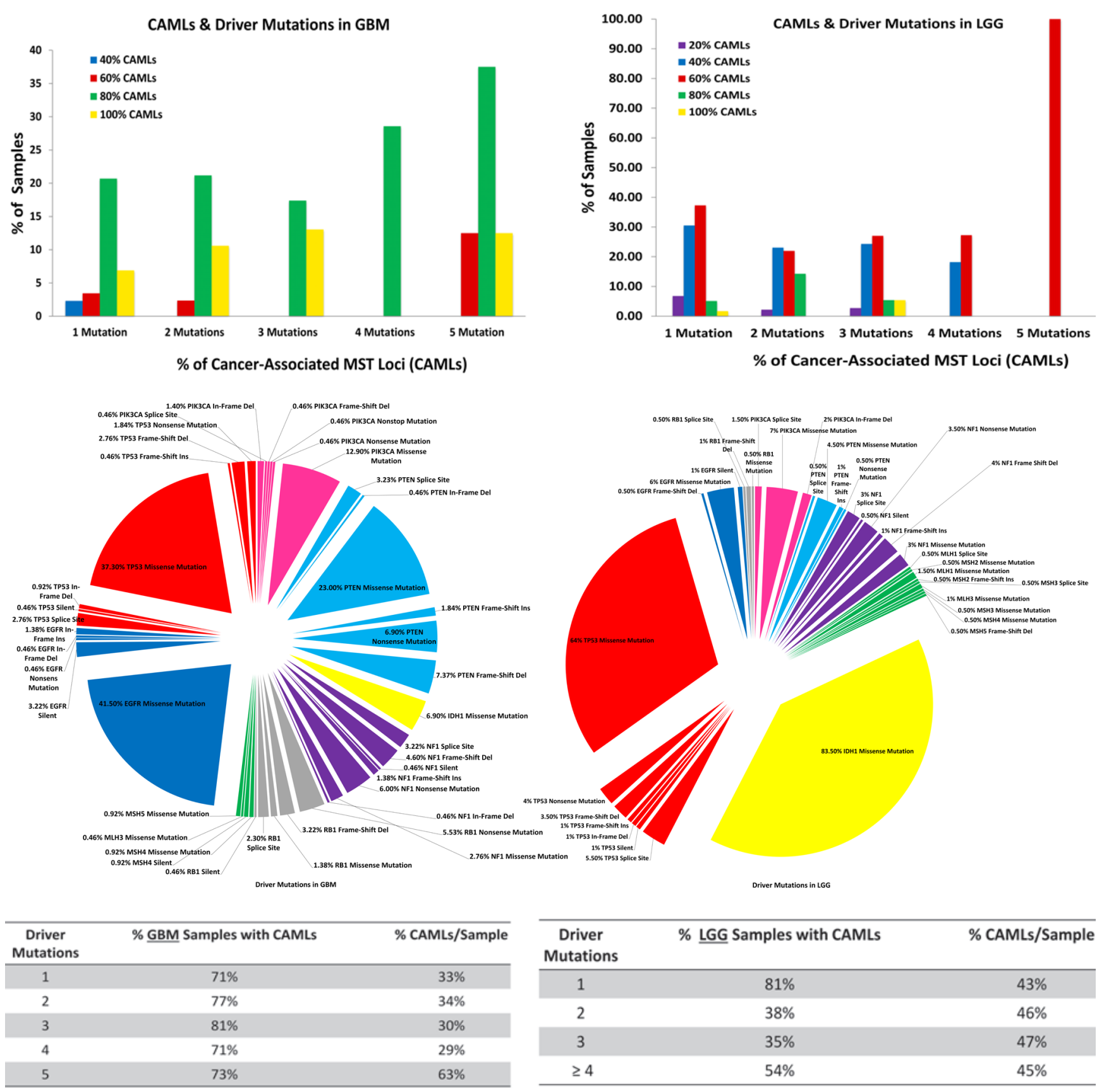

\begin{tabular}{ccc}
\hline $\begin{array}{c}\text { Driver } \\
\text { Mutations }\end{array}$ & \% GBM Samples with CAMLs & \% CAMLs/Sample \\
\hline 1 & $71 \%$ & $33 \%$ \\
\hline 2 & $77 \%$ & $34 \%$ \\
\hline 3 & $81 \%$ & $30 \%$ \\
\hline 4 & $71 \%$ & $29 \%$ \\
\hline 5 & $73 \%$ & $63 \%$ \\
\hline
\end{tabular}

\begin{tabular}{ccc}
\hline $\begin{array}{c}\text { Driver } \\
\text { Mutations }\end{array}$ & \% LGG Samples with CAMLs & \% CAMLs/Sample \\
\hline 1 & $81 \%$ & $43 \%$ \\
\hline 2 & $38 \%$ & $46 \%$ \\
\hline 3 & $35 \%$ & $47 \%$ \\
$\geq 4$ & $54 \%$ & $45 \%$ \\
\hline
\end{tabular}

Figure 3: A-C. Differences in Cancer-Associated Microsatellite Loci and Driver Mutations in GBM \& LGG. Frequently identified somatic mutations were quantitated from DNA sequencing samples from GBM $(n=216)$ and LGG $(n=200)$. Mutations in TP53, EGFR, RB1, NF1, PTEN, PIK3CA, IDH-1, and six mis-match repair genes were classified from sequencing samples and reported as a percentage of the total population for each glioma cohort. 3A: The percentage of samples with CAMLs (ranging from 20\%-100\%) and with up to 5 driver mutations (the maximum) are shown. 3B: A pie-chart was created to describe the distribution of mutations in those genes for GBM and LGG; mutations in p53, EGFR, and PTEN were frequently identified from GBM, while missense mutations in IDH-1 were the most common in LGG samples. Mutations in mis-match repair genes were infrequently identified from both GBM and LGG, regardless of the percentage of CAMLs identified in GBM or LGG. 3C: Samples with a given population of mutations (on average between 1-5) were examined to determine the percentage of GBM or LGG samples with CAMLs, and of these the percentage of CAMLs per sample. LGG samples contain more CAMLs compared with GBM, however, more GBM samples with CAMLs were identifiable. 
embedded in patient germlines that forecast tumor grade and discovered 29 signature CAMLs that distinguish LGG from GBM. Eleven of these loci were in LGG, CAML genotypes $(\mathrm{p} \leq 0.01)$ and 10 loci were found among significant LGG microsatellite (MST) genotypes. Two loci (9:52626-52640 and 2:91886031-91886042) are in the GBM signature and one locus was found amongst significant GBM genotypes (SSX2). Our next goal was to determine, if specific microsatellite variants could be further used to distinguish Grade II from GBM. Most Grade II samples (75\%) had between from $0 \%$ to $80 \%$ CAMLs, while $90 \%$ of GBM samples exhibited $100 \%$ CAMLs (Fig. 2B).

\section{Gene Ontologies \& Cell Functions Important to LGG \& GBM}

Molecular, cellular, and biological processes significantly $(\mathrm{p} \leq 0.1)$ associated with signature loci were analyzed using DAVID annotation tools[14]. From these annotations, we further evaluated individual genes and their potential roles in GBM biology, as described in detail in Tables S1, S2, and S3. Genes proximate to our CAMLs primarily contribute to RNA- processing and the ubiquitin proteasome system (UPS). Both of these cell functions are activated by interferon in viral pathogenesis, and both are important in identifying and degrading/correcting misfolded macromolecules (e.g. - RNA, DNA, and proteins). Additionally, malfunctions by the UPS are frequently linked to neuropathies and gliomas [15].

\section{Regulatory Elements near CAMLs in LGG \& GBM:}

We discovered $\mathrm{CpG}$ islands $(\leq 10 \mathrm{kB}$ from MST loci) and transcription factor binding sites (TFBS, $\leq 40$ bases of MST loci) near CAMLs. Ten MST loci from GBM and 9 LGG loci (with 3 loci shared between both glioma cohorts) were linked to $\mathrm{CpG}$ islands. Nine MST loci from GBM and 20 MST loci in LGG (with 2 loci shared between both populations) were located within or near TFBS (see Table S1). Illustrated in Figure S2 are the six helicase genes with CAMLs from the GBM signature: all six loci are within ENCODE methylation marker sites (H3KMe1 and H3KMe3), 5 of the loci are within transcription factor binding sites, and 2 are located within expressed sequence tags (EST).

\section{Correlation between CAMLs \& Gene Expression:}

We measured changes in gene expression and differences in transcripts for those genes with CAMLs, and RNA transcripts from glioma tumors (LGG and GBM) were compared with normal adult brain RNASeq data, previously described by Ameur, A. et. al, (Table 2) [16]. In our analysis from tumor versus "normal" germline samples, expression data demonstrated significant $(\mathrm{p} \leq$ 2.0-fold) down-regulation of $L N X 2$ and FGD6 and upregulation of CRISP1 in LGG. In GBM, a significant down-regulation of $S E M A 3 E$ and up-regulation of SLC44A4 were measured (see Tables S1 and S2).

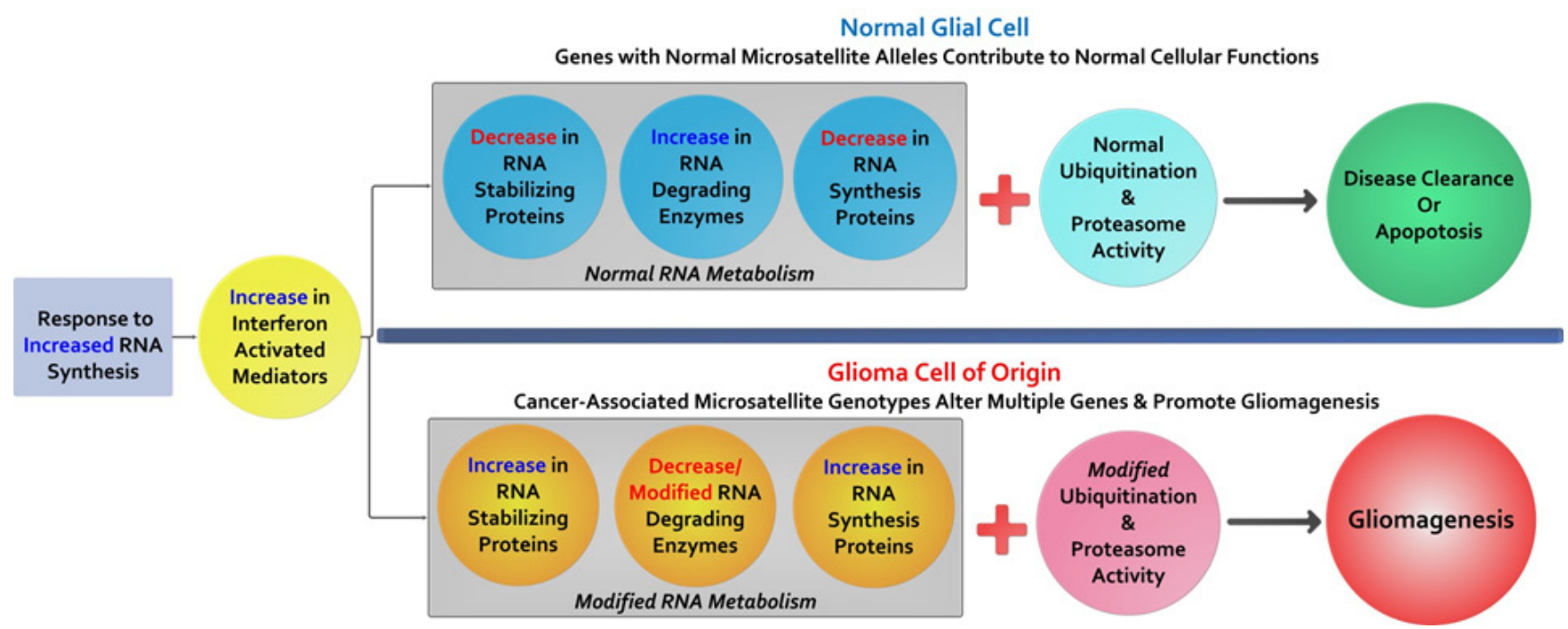

Figure 4: The potential contributions of cancer-associated microsatellite variants to gliomagenesis: Briefly outlined is a model to explain MST driven gliomagenesis. We hypothesize that these cancer-associated variants are a component of a glioma initiating cell population enriched for CAMLs that predict functional associations with RNA synthesis, RNA degradation, and protein degradation important to transcription complexes (spliceosomes, snRNPs, snoRNPs, and snRNA). As evidenced through our data, several helicases and E3 ubiquitin ligases that are transcriptionally up-regulated by interferon signaling harbor CAMLs, suggesting that a mosaic of genes with functions ascribed to known glioma-specific biology, may work differently in glioma cells with specific and variant microsatellite genotypes. These subtle yet compounding variants may produce gene-products which further intensify genomic instability causing greater genomic alterations (gene mutations, hypermethylation, chromosome loss/breakage, etc.) favoring cancer. 


\section{Correlation between CAMLs \& Glioma Associated Driver Mutations}

We analyzed TCGA gene mutation data (i.e. INDEL, frame shift, non-frame shift etc...) to identify connections between driver mutations loci (36 mutations in total identified in 7 genes and 6 mis-match repair genes (MMR)) closely identified with gliomas and the signature. Our data show that GBM samples contain $80 \%$ of the GBM CAML genotypes regardless of the number of driver mutations found within those samples and that the majority of LGG samples contain $60 \%$ of LGG CAML genotypes. However, an increase in CAMLs was identified in most samples with an average of 5 driver mutations, in both GBM and LGG. Interestingly, LGG samples with only 1 driver gene mutation (frequently, IDH-1) were the most likely population to demonstrate CAMLs, and on average $81 \%$ of these LGG samples had $43 \%$ of the LGG CAMLs (Fig. 3C). In general both GBM and LGG populations carry similar percentages of CAMLs, regardless of the number of driver mutations.

\section{DISCUSSION}

These data show microsatellite genotypes can differentiate cancer from non-cancer populations and also lower grade from GBM. Since these variant loci are identifiable in somatic DNA and are conserved in tumors lends support to the hypothesis that glioma initiating cell populations exist and are inherent to the individual and their disease.

Interestingly, we observe that a small number $\leq 3 \%$ of normal subjects have $100 \%$ of the GBM, CAML genotypes. This may be due to sample population age biases such that some individuals are as yet undiagnosed, or that our measure over-predicts given that the rate of gliomas in the general population is less than 3\%. Therefore risk screening may be valuable only in a subset of the population presenting with an abnormal MRI. Additionally, when we inspected samples with driver mutations, observed in gliomas, the relative percentage of CAMLs is similar in individuals with 1-4 mutations. However, for individuals with 5 mutations there appears to be an increase in identifiable CAMLs which may correspond to overall instability in the genome's of these individuals. The mix of glioma types in the LGG cohort may lend to smaller populations of LGG DNA sequences with identifiable CAMLs compared with GBM (as observed in Fig. 2B, Fig 3C, and FigS3) which suggests that distinguishable and conserved MST loci may further differentiate glioma phenotypes and may be tumor specific markers. Circulating disease markers for tissue-specific cancers are progressively becoming more detectible; however diagnosing primary brain cancer markers outside of brain tissue remains poor[17]. Therefore, testing for these CAMLs from blood samples could provide a potential supplemental diagnostic resource to identify the most common and dangerous adult brain-specific cancers.

Since some microsatellites are associated with DNA 'fragile sites', locations within chromatin susceptible to constrictions or break-points that are linked to cancers and neuro-developmental disorders, we analyzed our MST loci to determine which are located in these regions, as a possible mechanism for tumor potentiation [18, 19]. $B R W D 2$, found in our GBM signature, is located at a break-point on chromosome 10 and allelic deletions within 10p, 10q 25-26, and 19q 13.3-13.4 are the most common alterations in glial tumors[20,21]. Given the location of the breaks, $B R W D 2$ is considered a candidate tumor suppressor; through our analysis we identified $80 \%$ of GBM patients versus $50 \%$ of "normal" individuals have a CAML genotype at BRWD2. Interestingly, FGFR2 and $B R W D 2$, both genes included in the GBM signature, are an oncogene and tumor suppressor pair located at a recombination locus in which deletion of exon 21 of $F G F R 2$ results in the exclusion of BRWD2 (the tumor suppressor) and amplification of FGFR2; thus intronic CAMLs harbored by these genes could be biological indicators for tumorigenic activity. Additionally, loss of $10 \mathrm{p}$ is found in $47 \%$ of GBMs while $10 \mathrm{q}$ loss is found in $70 \%$ of primary and $63 \%$ of secondary GBMs[20]. In our GBM signature, we identified 4 loci in $\mathrm{Ch} 10$ at FGFR2, BRWD2 (WDR11), GLUD1, and NRP1; none were identified in the LGG signature.

In the original account of 'the two-hit hypotheses' for somatic retinoblastoma, the first hit was an inherited mutation in $R b$, rendering individuals more susceptible to the disease later in life. Our data suggests the 'first hit' may also be somatic microsatellite instability which then increases the sensitivity to, and probability of, greater genomic aberrance theoretically by stress factors such as environment, inflammation or age leading to the "second hit'[22-24]. These tentatively 'predisposed' variants may highlight a connection between cancer-associated microsatellites and developmental and cell cycle genes notably mutated in cancer (such as those identified in our study: SRC, NPAT and $C B L$ ); thus, these MST variants may be additive to gene mutations (including, SNPs) that contribute to cancer.

More recently, mutable genes commonly identified in GBM patients post chemotherapy and radiation treatment were discovered, and included RYR1 [25]. RYR1 expression is notably down-regulated in GBM; our GBM data demonstrates CAML genotypes unique to RYRI with transcripts and isoforms also unique to GBM. Thus, CAML loci at genes such as RYR 1 may render them to be more susceptible to mutation (for example from stresses associated with treatment) and may be valuable indicators for potential treatment outcomes. Additionally, this further supports the possibility of tumor initiating cells with irregular genetic variations that generate disease relative 
to an underlying combination and abundance of affected microsatellite loci.

The aberrant alteration of six helicase genes in GBM suggests that genes important to microsatellite identification and correction, along with transcription and RNA synthesis are themselves modified with MST variants. Previous studies have demonstrated that in addition to mis-match repair genes, helicases (notably RecQ family DNA helicases [26]) are important in DNA damage repair and DNA/RNA metabolism[27]. In our data we observed minimal MMR gene coding SNP mutations in both GBM and LGG tumors (see, Fig 3B) but found CAMLs significantly associated with helicases, suggesting modifications to these genes may also be important to genomic instability in glioma or that there may be more MMR genes that remain to be discovered. As such, one mechanism may be that GBM tumors produce atypical RNA. This idea is further supported by the enrichment of MST variant loci in helicase genes activated through interferon; interferon can initiate helicases and ubiquitin ligases to degrade viral RNAs and other dsRNAs (see Table S3), a process which could be interrupted if these helicases are affected by MST. As described in Table 2, we identified multiple genes in which a novel RNA isoform was the abundant transcript for several GBM genes with CAMLs, including DICER1 and POLQ, both genes associated with RNA and DNA modification and metabolism.

With the abundance of helicase and UPS associated genes in our glioma signatures, another cancer promoting scenario may be introduced through changes to geneproducts that compose spliceosome complexes (snRNA, snRNP, or snoRNP); through these genetic modifications, alternatively spliced RNAs may support spliceosomeassociated proteins differently, which may further modify mature RNAs. A third mechanism may be modifications to ubiquitin proteasome system proteins (ligases and ubiquitin complex proteins) which could alter protein degradation or signal transduction. Supporting these two hypotheses we identified functionally associated genes with CAMLS, including: DDX60 and TRIM25; SPOPL and BRMS1L; FGFR2, BRWD2 and CBL; SEMA3E and $N R P 1$. Other functional associations important to changes in RNA transcription included, SLC44A4 transcript isoforms uniquely identified in GBM (and with expression significantly greater than normal tissue). Similar results were observed for $L N X 2$ in $\mathrm{LGG}$ supporting our hypothesis that CAMLs may modify RNA transcription and expression.

Additional important biological changes observed and linked to CAMLs included significantly decreased expression of SEMA3E (a GBM signature locus) compared with normal samples; as previously noted SEMA3 competes with $V E G F-165$ for NRP1 (a GBM signature locus) and suppresses angiogenesis [28]. Therefore, decreased expression of SEMAs in GBM suggests that these microsatellite variants could contribute to glioma angiogenesis. Several genes, including the EVC transcript were identified in GBM and LGG but not in normal brain tissue, and in GBM the novel isoform was the common transcript; expression data suggests that $E V C$ is expressed more in GBM and LGG tumors when compared with normal germline expression, although these data did not pass our stringent 2-fold difference in expression (GBM =1.68; $\mathrm{LGG}=1.97)$.

Since these loci can be identified from germline (non-tumor) DNA, it further suggests that there is a tumor initiating cell population in which developmental cell signaling and patterning pathways may result in aberrant differentiation and proliferation due to an underlying transcriptional landscape determined by cancer-associated MST variants. Therefore, we further hypothesize that DNA microsatellite variability underlies an adaptability that is conserved in all cancers and can be investigated by determining the frequency of microsatellite variability in those genes (1) that are essential for cancer cell survival (and conserved across a cancer type) (2) contribute intermittently - to cancer cell phenotypes like metastasis, heterogeneity, or aggressiveness, and (3) are tissuespecific, associated with only one type of tumor or tissue origin. If so, we can predict that specific microsatellite instability contributes to cancer-specific genomics and may occur during embryogenesis, which has also been predicted in other MST associated diseases, including Huntington's disease and Fragile X syndrome[13]. Thus, these markers may be valuable to screen risk of occurrence or for treatment decisions in newly diagnosed cancer patients.

\section{MATERIALS \& METHODS}

\section{Microsatellite genotyping}

Exome sequencing data, from Illumina HiSeq sequencing machines were obtained from The Cancer Genome Atlas (TCGA) and the 1000 Genomes Project $(1 \mathrm{kGP})$. These sequences were aligned to the human reference genome using BWA, by their respective projects, and re-alignment followed with microsatellite loci and variant identification by methods and software established in our laboratory [29-31]. Only loci with sequencing reads with $15 \mathrm{x}$ or greater depth of coverage were used to identify possible informative loci. A distribution of alleles for the affected (TCGA) and unaffected (1kGP) cohorts was then generated for each locus. An allele is defined by a genomic locus with a specific microsatellite repeat and nucleotide sequence length, in each sample a pair of alleles was identified and each allelic pair was then defined as a genotype. The genotype most prevalent from a distribution of genotypes was identified in $1 \mathrm{kGP}$ 
samples; this genotype was defined as the consensus sequence or predominant genotype (if more than a pair of alleles was identified for a locus that locus in the particular sample was not used). Similar to the $1 \mathrm{kGP}$ samples, LGG and GBM samples were analyzed for genotypes from the same genomic loci, loci significantly different from the consensus (predominant genotype) in one population compared with the second population were identified. The statistically significant genotypes were determined from data adjusted for false discovery rate (FDR), using a two-sided Fisher's p- test and Benjamini-Hochberg correction; relative risk (RR) was calculated for each locus and loci with a $\mathrm{P} \leq 0.01$ were considered significant. More specifically, an $\mathrm{R}$ script computed the $\mathrm{p}$-value for each locus using the two sided fisher-test function. The Benjamini-Hochberg cut-off was selected as approximately $0.01 \%$ (computed as the FDR $<1 / \mathrm{X}$ (where $\mathrm{x}$ is the total number of loci with $\mathrm{p}$-value $<1$ for the signature)) to make it unlikely that any locus is a false positive from our data set. Those genotypes, although individually significant and informative, were also assembled into a 'signature' or 'cancer-associated' informative loci set which together increase the statistical significance across all samples. Sequences included 390 $(\mathrm{n}=249$ female; $\mathrm{n}=141$ male) normal/healthy samples from the $1 \mathrm{kGP}$, GBM germline $(\mathrm{n}=252)$, GBM tumor $(n=139)$, LGG germline $(n=136)$, and LGG tumor $(n=158)$ sequencing samples (dbGAP Study Accession: phs000178. v8.p7) [32] through the Cancer Genomics Hub (CGHub). Microsatellite genotypes were identified through a software system developed in our laboratory [30, 31]. These samples, like all others, were processed to remove any reads that did not meet the QC thresholds required in the $1 \mathrm{kGP}$ [33]

\section{Creation of microsatellite target set}

We produced a set of over 850,000 microsatellite loci which have flanking sequences unique in the human genome. Initially a set of over a million microsatellites was found in the human genome (NCBI36/hg18) using Tandem Repeats Finder (TRF) [34], with previously established parameters. A series of filters are in place to remove tandem repeat sequences previously identified through RepeatMasker in the flanking sequences of our loci, microsatellites shorter than 12 nucleotides in regions other than exons for which we allowed a minimum length of 10 nucleotides, those containing SNPs, indels, or SNP variations that account for variability greater than $10 \%$ of the locus lengths (Repeat Masker Ref. Smit AFA; HR Green). A custom Perl script was written to filter repeat sequences lacking unique flanking regions (10 base flanking sequences were chosen because Illumina reads are 100 bases in length, respectively); filtered sequences were screened based on corresponding flanking sequences to the reference genome (microsatellites with flanking sequences within 200 bases of each other and 5 bases of the repeat in between were also excluded). Vetted microsatellite loci are described with genomic location and gene associations using RefSeq data obtained through the UCSC Genome Table Browser [35] .

\section{Identifying repeat lengths using microsatellite- based genotyping}

Reads are mapped to the reference genome using BWA or BWA-sw (for long reads; LS454) [36]. A custom Perl script with SAMTOOLS [37] filters for reads with MST loci, to assure the span of the MST sequence is captured, a 5 base flanking sequence on either side of the read are tested, this process is to validate BWA aligned sequences. Reads with identical repeat sequences are binned; separating reads with different SNPs. SNPs contained in microsatellites were ignored for this study.

Accuracy estimations of our microsatellite-based genotyping method: Using Sanger sequencing and data from HapMap, we were able to validate $96.5 \%$ of a subset of 85 non-synonymous variations composed of repeat length variations and SNPs contained in microsatellites. The novel variants we validated using Sanger sequencing were submitted under the lab handle SGARNER and are available on-line in the latest release of dbSNP. All microsatellite-based genotyping calls from these studies were made publically available at MicrosatDB (http:// discovery.vbi.vt.edu/MicrosatDB/) [31].

\section{Microsatellite identification restrictions for population-based statistics}

To increase uniformity of coverage and genotyping rates across samples sequenced at different times with different methods by different studies, we required at least 15,000 microsatellite loci to be called per sample for inclusion in this study. This filtered out one $1 \mathrm{kGP}-\mathrm{F}$ sample and $2351 \mathrm{kGP}-\mathrm{M}$ samples. From the TCGA samples, this filtered out 23 LGG germline samples, 57 LGG tumor samples, 238 GBM germline samples, and 254 GBM tumor samples. The large number of GBM samples filtered is expected as these samples were sequenced much earlier by TCGA than the LGG samples. So we would expect lower coverage and shorter reads. Only those loci with at least $15 x$ coverage are considered "callable" in a given sample (healthy or cancer genomes). A locus had to be called in a minimum of 10 exomes to be included in the genotype distribution comparison analysis and to remove loci which may be called at insufficient frequency in one of the two data sets. 


\section{Alternative splicing}

We processed 339 GBM tumors, 268 LGG tumors, and 57 LGG germline RNAseq Illumina data sets with Cufflinks [38] by using the CuffCompare function to identify possibly alternatively spliced transcripts. These samples had been aligned by TCGA to the reference using TopHat[39]. For each transcript for each sample, we determined it was possibly alternatively spliced if one of the transcripts called by CuffCompare was not a complete match of the intron chain. Additionally, RNAseq data was downloaded for two normal brain tissue samples, adult [16]. These RNAseq SOLiD fastq read files where reformatted into standard color-space paired files and then run through TopHat using the default parameters, except for indicating the color-space type, as was done by the TCGA to align the GBM/LGG samples. These samples were then processed using Cufflinks to identify possibly alternatively spliced transcripts.

\section{CpG Island and TFBS Analysis}

Locations of $\mathrm{CpG}$ islands and transcription factor binding sites associated with the human reference genome were downloaded from the UCSC Genome Browser [35]. A Perl script was written to determine the distance of each microsatellite loci from the nearest $\mathrm{CpG}$ island and transcription factor binding site. Methylation data was downloaded for the samples from TCGA. This data provided the $\mathrm{CpG}$ islands and corresponding gene with respect to each methylation site. A Perl script was written to compute methylation averages for each site for samples with and without CAML signature loci for the corresponding gene.

\section{Identification of Driver Mutations in GBM \& LGG Samples}

Variant calls for the GBM and LGG samples were downloaded from TCGA. The driver mutations and MMR genes of interest (IDH1, PTEN, PIK3CA, RB1, MSH2, MSH3, MSH4, MSH5, MSF6, MLH1, and MLH2) were analyzed with respect to the CAML status of the samples.

\section{ACKNOWLEDGEMENTS}

This work was funded by the Medical Informatics and Systems Division directors fund to Dr. Garner at VBI/ Virginia Tech and computation was partially supported by an NSF grant. We would like to thank The Cancer Genome Atlas and The 1000 Genomes Project for their generous distribution of genomic data analyzed in this study. We thank the system administrators in the VBI computational core (Michael Snow, Dominik Borkowski, David Bynum,
Douglas McMaster, Jeremy Johnson, and Vedavyas Duggirala) for technical support.

\section{COMPETING INTERESTS}

Dr. Harold R. Garner is a co-owner of Genomeon, L.L.C., a startup company which has entered into an exclusive licensing agreement with Virginia Tech for the microsatellite loci and the microsatellite identification software described in this report. Genomeon played no role or influenced the direction of this research.

\section{AUTHOR'S CONTRIBUTIONS}

EK and LJM made substantial contributions to the analyses, data/evidence collection, visualization/ data presentation, and conception/design of the study. EK wrote the initial draft of the manuscript and made substantial contributions related to data interpretation. LJM performed all programming/software development including running code and compiling statistics on large data sets. BRD contributed some of the conception/design of the study and critical revising of the manuscript. XW and $\mathrm{HZ}$ contributed to some of the statistical analyses. JHB contributed some of the analyses specific to somatic mutations. HRG made substantial intellectual contributions to the conception/design of the study and manuscript development.

\section{DESCRIPTION of ADDITIONAL DATA FILES}

I.The following additional data are available with the online version of this paper. Data file 1 titled "Supplementary Information.doc") contains figures and tables associated with the following:

a.ROC analysis

b.CpG and TFBS loci

c.RNA expression

d.Ontologies/gene functions

e.LGG sub-group

II.Data file 2 contains all computational analysis summarized in an 'Excel' file, titled "Supplementary Data_Glioma MetaData.xlsx'

\section{REFERENCES}

1. Siegel R, Naishadham D and Jemal A. Cancer statistics, 2012. CA: A Cancer Journal for Clinicians. 2012; 62(1):1029.

2. Furnari FB, Fenton T, Bachoo RM, Mukasa A, Stommel JM, Stegh A, Hahn WC, Ligon KL, Louis DN, Brennan C, Chin L, DePinho RA and Cavenee WK. Malignant astrocytic glioma: genetics, biology, and paths to treatment. Genes \& development. 2007; 21(21):2683-2710. 
3. Stupp R, Mason WP, van den Bent MJ, Weller M, Fisher B, Taphoorn MJ, Belanger K, Brandes AA, Marosi C, Bogdahn U, Curschmann J, Janzer RC, Ludwin SK, Gorlia T, Allgeier A, Lacombe D, et al. Radiotherapy plus concomitant and adjuvant temozolomide for glioblastoma. The New England journal of medicine. 2005; 352(10):987996.

4. Persson AI, Petritsch C, Swartling FJ, Itsara M, Sim FJ, Auvergne R, Goldenberg DD, Vandenberg SR, Nguyen KN, Yakovenko S, Ayers-Ringler J, Nishiyama A, Stallcup WB, Berger MS, Bergers G, McKnight TR, et al. Nonstem cell origin for oligodendroglioma. Cancer cell. 2010; 18(6):669-682.

5. Liu C, Sage JC, Miller MR, Verhaak RG, Hippenmeyer S, Vogel H, Foreman O, Bronson RT, Nishiyama A, Luo L and Zong $\mathrm{H}$. Mosaic analysis with double markers reveals tumor cell of origin in glioma. Cell. 2011; 146(2):209-221.

6. Phillips HS, Kharbanda S, Chen R, Forrest WF, Soriano RH, Wu TD, Misra A, Nigro JM, Colman H, Soroceanu L, Williams PM, Modrusan Z, Feuerstein BG and Aldape K. Molecular subclasses of high-grade glioma predict prognosis, delineate a pattern of disease progression, and resemble stages in neurogenesis. Cancer cell. 2006; 9(3):157-173.

7. Parsons DW, Jones S, Zhang X, Lin JC, Leary RJ, Angenendt P, Mankoo P, Carter H, Siu IM, Gallia GL, Olivi A, McLendon R, Rasheed BA, Keir S, Nikolskaya T, Nikolsky Y, et al. An integrated genomic analysis of human glioblastoma multiforme. Science. 2008; 321(5897):18071812.

8. Baysan M, Bozdag S, Cam MC, Kotliarova S, Ahn S, Walling J, Killian JK, Stevenson H, Meltzer P and Fine HA. G-cimp status prediction of glioblastoma samples using mRNA expression data. PloS one. 2012; 7(11):6.

9. Comprehensive genomic characterization defines human glioblastoma genes and core pathways. Nature. 2008; 455(7216):1061-1068.

10. Gemayel R, Vinces MD, Legendre M and Verstrepen KJ. Variable tandem repeats accelerate evolution of coding and regulatory sequences. Annu Rev Genet. 2010; 44:445-477.

11. Jasinska A and Krzyzosiak WJ. Repetitive sequences that shape the human transcriptome. FEBS letters. 2004; 567(1):136-141.

12. Richard GF, Kerrest A and Dujon B. Comparative genomics and molecular dynamics of DNA repeats in eukaryotes. Microbiol Mol Biol Rev. 2008; 72(4):686-727.

13. McMurray CT. Mechanisms of trinucleotide repeat instability during human development. Nature reviews Genetics. 2010; 11(11):786-799.

14. Dennis G, Jr., Sherman BT, Hosack DA, Yang J, Gao W, Lane HC and Lempicki RA. DAVID: Database for Annotation, Visualization, and Integrated Discovery. Genome biology. 2003; 4(5):3.

15. Lehman NL. The ubiquitin proteasome system in neuropathology. Acta neuropathologica. 2009; 118(3):329347.

16. Ameur A, Zaghlool A, Halvardson J, Wetterbom A, Gyllensten U, Cavelier L and Feuk L. Total RNA sequencing reveals nascent transcription and widespread co-transcriptional splicing in the human brain. Nature structural \& molecular biology. 2011; 18(12):1435-1440.

17. Holdhoff M, Yovino SG, Boadu O and Grossman SA. Blood-based biomarkers for malignant gliomas. Journal of neuro-oncology. 2013; 113(3):345-352.

18. Katoh M. FGFR2 and WDR11 are neighboring oncogene and tumor suppressor gene on human chromosome 10q26. International journal of oncology. 2003; 22(5):1155-1159.

19. Yudkin D, Hayward B, Aladjem MI, Kumari D and Usdin K. Chromosome fragility and the abnormal replication of the FMR1 locus in Fragile X syndrome. Human molecular genetics. 2014; 12:12.

20. Ohgaki $\mathrm{H}$ and Kleihues $\mathrm{P}$. The definition of primary and secondary glioblastoma. Clinical cancer research : an official journal of the American Association for Cancer Research. 2013; 19(4):764-772.

21. Chernova OB, Hunyadi A, Malaj E, Pan H, Crooks C, Roe $\mathrm{B}$ and Cowell JK. A novel member of the WD-repeat gene family, WDR11, maps to the 10q26 region and is disrupted by a chromosome translocation in human glioblastoma cells. Oncogene. 2001; 20(38):5378-5392.

22. Vogelstein B, Papadopoulos N, Velculescu VE, Zhou S, Diaz LA, Jr. and Kinzler KW. Cancer genome landscapes. Science. 2013; 339(6127):1546-1558.

23. Caruso C, Lio D, Cavallone L and Franceschi C. Aging, longevity, inflammation, and cancer. Annals of the New York Academy of Sciences. 2004:1-13.

24. Garagnani P, Pirazzini C and Franceschi C. Colorectal cancer microenvironment: among nutrition, gut microbiota, inflammation and epigenetics. Curr Pharm Des. 2013; 19(4):765-778.

25. Johnson BE, Mazor T, Hong C, Barnes M, Aihara K, McLean CY, Fouse SD, Yamamoto S, Ueda H, Tatsuno K, Asthana S, Jalbert LE, Nelson SJ, Bollen AW, Gustafson WC, Charron E, et al. Mutational analysis reveals the origin and therapy-driven evolution of recurrent glioma. Science. 2014; 343(6167):189-193.

26. Gaymes TJ, Mohamedali AM, Patterson M, Matto N, Smith A, Kulasekararaj A, Chelliah R, Curtin N, Farzaneh F, Shall S and Mufti GJ. Microsatellite instability induced mutations in DNA repair genes CtIP and MRE11 confer hypersensitivity to poly (ADP-ribose) polymerase inhibitors in myeloid malignancies. Haematologica. 2013; 98(9):13971406.

27. Song L, Yuan F and Zhang Y. Does a helicase activity help mismatch repair in eukaryotes? IUBMB life. 2010; 62(7):548-553.

28. Karayan-Tapon L, Wager M, Guilhot J, Levillain P, Marquant C, Clarhaut J, Potiron V and Roche J. 
Semaphorin, neuropilin and VEGF expression in glial tumours: SEMA3G, a prognostic marker? British journal of cancer. 2008; 99(7):1153-1160.

29. Galindo CL, McIver LJ, Tae H, McCormick JF, Skinner MA, Hoeschele I, Lewis CM, Minna JD, Boothman DA and Garner HR. Sporadic breast cancer patients' germline DNA exhibit an AT-rich microsatellite signature. Genes Chromosomes Cancer. 2011; 50(4):275-283.

30. McIver LJ, Fondon JW, 3rd, Skinner MA and Garner HR. Evaluation of microsatellite variation in the 1000 Genomes Project pilot studies is indicative of the quality and utility of the raw data and alignments. Genomics. 2011; 97(4):193199.

31. McIver LJ, McCormick JF, Martin A, Fondon JW, 3rd and Garner HR. Population-scale analysis of human microsatellites reveals novel sources of exonic variation. Gene. 2013; 516(2):328-334.

32. Cancer Genome Atlas N. Comprehensive molecular portraits of human breast tumours. Nature. 2012; 490(7418):61-70.

33. Genomes Project C, Abecasis GR, Auton A, Brooks LD, DePristo MA, Durbin RM, Handsaker RE, Kang HM, Marth GT and McVean GA. An integrated map of genetic variation from 1,092 human genomes. Nature. 2012; 491(7422):56-65.

34. Benson G. Tandem repeats finder: a program to analyze DNA sequences. Nucleic acids research. 1999; 27(2):573580.

35. Karolchik D, Hinrichs AS, Furey TS, Roskin KM, Sugnet CW, Haussler D and Kent WJ. The UCSC Table Browser data retrieval tool. Nucleic acids research. 2004; 32(Database issue):D493-496.

36. Li H and Durbin R. Fast and accurate short read alignment with Burrows-Wheeler transform. Bioinformatics. 2009; 25(14):1754-1760.

37. Li H, Handsaker B, Wysoker A, Fennell T, Ruan J, Homer N, Marth G, Abecasis G, Durbin R and Genome Project Data Processing S. The Sequence Alignment/Map format and SAMtools. Bioinformatics. 2009; 25(16):2078-2079.

38. Trapnell C, Williams BA, Pertea G, Mortazavi A, Kwan G, van Baren MJ, Salzberg SL, Wold BJ and Pachter L. Transcript assembly and quantification by RNA-Seq reveals unannotated transcripts and isoform switching during cell differentiation. Nature biotechnology. 2010; 28(5):511-515.

39. Trapnell C, Pachter L and Salzberg SL. TopHat: discovering splice junctions with RNA-Seq. Bioinformatics. 2009; 25(9):1105-1111.

40. Shafqat S, Hedley-Whyte ET and Henson JW. Agedependent rate of anaplastic transformation in low-grade astrocytoma. Neurology. 1999; 52(4):867-869. 